\title{
Repeated doses of porcine secretin did not improve symptoms, language, or cognitive functioning in children with autism or autism spectrum disorder
}

Roberts W, Weaver L, Brian J, et al. Repeated doses of porcine secretin in the treatment of autism: a randomized, placebo-controlled trial. Pediatrics 2001 May;107:E71.

QUESTION: In children with autism or autism spectrum disorder (ASD), are repeated doses of porcine secretin more effective than placebo for improving symptoms, language, and cognitive functioning?

\section{Design}

Randomised \{allocation concealed*\} $\uparrow$, blinded (patients, parents, and outcome assessors)*, placebo controlled trial with up to 10 weeks of follow up.

\section{Setting}

Hospital for Sick Children, University of Toronto, Canada.

\section{Patients}

68 children (age range $2-7 y, 84 \%$ boys) who had autism or ASD according to the Autism Diagnostic InterviewRevised (ADI-R) and the Autism Diagnostic Observation Scale-Generic (ADOS-G) and had previously received a diagnosis of autism or pervasive developmental disorder based on DSM-IV. 94\% of children completed the study.

\section{Intervention}

34 children were allocated to each of the porcine secretin $(2 \mathrm{ml} / \mathrm{kg})$ and placebo groups. The children received 2 intravenous injections of secretin or placebo 6 weeks apart. Before each injection, the children fasted for 3 hours and received a test dose for allergic response.

\section{Main outcome measures}

Assessment of symptoms of autism (Autism Behaviour Checklist and Treatment/Side Effect Behaviour Scale by parents and ADOS-G by clinicians), language (Preschool Language Scale III [PLS-3]) and ADOS-G), visual spatial attention task, and physiological and/or gastrointestinal (GI) symptoms (parent questionnaires). Cognitive function (Leiter International Performance Scale-Revised and Vineland Adaptive Behavior Scales) was assessed at baseline and final follow-up.

\section{Main results}

The porcine secretin and placebo groups did not differ for all measures of autistic symptoms, cognition, and language (all $p$ Values $>0.05$ ). Subgroup analysis based on cognitive level, presence or absence of GI symptoms, or history of regression showed no treatment effect of porcine secretin when compared with placebo (all $\mathrm{p}$ Values $>0.05$ ). The study had $80 \%$ power to detect an overall moderate effect on the PLS-3 at the 5\% level of significance.

\section{Conclusions}

In children with autism or autism spectrum disorder, 2 doses of porcine secretin were not more effective than placebo for improving symptoms, language, and cognitive functioning.

*See glossary.

$\dagger$ Information provided by author.
Source of funding: no external funding.

For correspondence: Dr W Roberts, Child Development Centre Hospital for Sick Children, 555

University Avenue, Toronto, Ontario $\mathrm{M} 5 \mathrm{C}$ 1X8, Canada. Fax +1 4168137437.

\section{COMMENTARY}

The aetiology of many of the ASDs is poorly understood. As a result many undocumented treatments have come into popular use. Secretin is an example of a treatment in use without evidence-based studies showing its efficacy. Thousands of doses of secretin have been administered to children with ASD, despite growing research documentation of its ineffectiveness to change the symptoms of autism. This trial by Roberts et al contributes to the existing body of knowledge. It addresses common criticisms for negative effects in previous studies by using a multiple dose regimen and assessing GI symptoms and severity of autism.

This study used state of the art tools for diagnosis and outcome measurement. When the study sample was stratified by GI symptoms or severity of autism, no differences were seen between groups. This deals with the contention that children with GI symptoms or more severe autism are more likely to respond. ${ }^{1}$

Both groups showed objective and subjective improvement across several domains of development, which may be attributed to the natural history of the disorder or the placebo effect. ${ }^{2}$ Most importantly, the amount of improvement did not distinguish the 2 groups. This highlights the need for a control group and objective (and blinded) measures of change.

Since the initial case report in 1998, over 300 children have completed double blind placebo controlled trials, failing to corroborate the observations of behavioural changes following secretin. ${ }^{3}{ }^{4}$ With this information, clinicians who are confronted with families pursuing all potential cures should feel more confident to guide them away from the unproven theoretical treatments back to the standard educational, communication, developmental, and behavioural interventions.

Susan E Levy, MD

The Children's Hospital of Philadelphia Philadelphia, Pennsylvania, USA

Horvath K. Secretin treatment for autism. N Engl J Med 2000;342:1216.

2 Sandler AD, Bodfish JW. Placebo effects in autism: lessons from secretin. J Dev Behav Pediatr 2000;21:347-50.

3 Dunn-Geier J, Ho HH, Auersperg E, et al. Effect of secretin on children with autism: a randomized controlled trial. Dev Med Child Neurol 2000;42:796-802.

4 Coniglio SJ, Lewis JD, Lang C, et al. A randomized, double-blind, placebo-controlled trial of single-dose intravenous secretin as treatment for children with autism. J Pediatr 2001;138:649-55. 